\title{
Power enhancement via an ion-channel in a Raman free-electron laser
}

\author{
Xiaoli Huang ${ }^{1,2}$ and Shi-Chang Zhang ${ }^{3}$ \\ ${ }^{1}$ School of Electrical Engineering and Electronic Information, Xihua University, Chengdu SC610039, \\ People's Republic of China \\ 2 Department of Physics, University of Fribourg, Fribourg-1700, Switzerland \\ ${ }^{3}$ School of Information Science and Technology, Southwest Jiaotong University, Campus Mail Box 50, \\ Chengdu 610031, People's Republic of China \\ E-mail: sczhang@home.swjtu.edu.cn
}

\begin{abstract}
As a high-power and high-efficiency coherent radiation source, the free-electron laser extends to two unique spectra: the region in the ultra-violet, $\mathrm{x}$ - and $\gamma$-rays, and the range in the millimeter- and terahertz-waves. Based on credible simulations, it is shown that the nonlinear power of a Raman free-electron laser in a millimeter wave range goes up and gets an increase of at least $9 \%$ when an ion-channel is applied to the beam-wave interaction chamber. A physical explanation is that the ion-channel weakens the velocities spread over the electron beam and, therefore, improves the electron-beam transportation quality and the beam-wave interaction.
\end{abstract}

Keywords: free-electron laser, power enhancement, ion-channel, relativistic electron beam

\section{Introduction}

In recent years, free-electron lasers (FELs) have been demonstrated to be high-power and high-efficiency coherent radiation sources, and have found applications in two unique spectra: one is in the ultra-violet, $x$-ray and $\gamma$-ray regions [1-9], and the other is in the millimeter- and terahertz-wave ranges [10-18].

As a free-electron laser in the millimeter- and terahertzwave ranges operates in a Raman-type regime, a high current of the electron beam with mildly relativistic energy is always employed to achieve an output power that is as high as possible. Because the electron beam is formed by individual, free electrons; the repulsion force among them must be produced by their self-field, and hence has an effect to diverge the beam itself. Divergence of the electron beam becomes serious when it has a large current density. In practice, therefore, an axial magnetic field is always used to guide the electron motion to be confined on approximate Larmor orbits [19-22]. An alternative way to prevent the electron beam from divergence is the application of an ionchannel guiding, which compromises the repulsion among the free electrons [23-25]. Compared to other plasmaloaded radio frequency devices, where external magnets are used, the advantage of ion-channel guiding, instead of the guide magnetic field, is that it cuts off the facility manufacture and operation expenses. Since the proof-of-principle experiments of the so-called ion-channel guiding FELs were made [26, 27], a lot of papers have been devoted to this aspect, discussing the ion-hose instability, harmonic generation, saturation mechanism, two-beam radiation, focusing peculiarity, electron's equilibrium orbits, motion chaos, beam temperature effect, etc [28-41].

For this paper, we concentrated our attention on the power enhancement in a Raman free-electron laser in millimeterand terahertz-wave ranges by making use of an adjusted ionchannel parameter. We present a physical explanation for the mechanism. In the following section, a general consideration is described for the beam-wave interaction in a Raman freeelectron laser with the application of an ion-channel. Then nonlinear simulations are carried out in sections 3 and 4 to show the enhancement of the nonlinear power by an adjusted ion-channel, and a physical explanation to the mechanism is presented. Finally, conclusions are drawn in section 5. 


\section{Nonlinear consideration}

Previously, nonlinear models of a Raman free-electron laser had various considerations (for example, see [16, 19]). Before describing the model of a Raman FEL with an ion-channel, it should be mentioned that the situation discussed in this paper is somewhat different from that in an ion-channel laser [25]. In an ion-channel laser, the ion hose is employed to replace the guide magnetic field, so as to confine the relativistic electron beam. Therefore, the ion-channel density in an ion-channel laser must be quite large to keep the ion-hose field strong enough to focus the relativistic electron beam. As was pointed out in [25], this requires the ion-channel density $n_{\text {ion }} / n_{b} \gg 1 / \gamma^{2}$. In an ion-channel laser, the ion-hose instability should be taken into account, since the ion-hose field is so strong. In the situation considered in this paper, however, the guide magnetic field is still presented to confine the relativistic electron beam, while the ion-channel is mild and just plays a role of an adjusted parameter to enhance the laser power. Therefore, the condition $n_{i o n} / n_{b} \gg 1 / \gamma^{2}$ is no longer necessary. Because of this reason, in our model, the ion-hose instability as well as the plasma oscillation could be negligible, and we ignore these effects.

Now, consider the configuration in a cylindrical waveguide with a 3D helical wiggler and an axial guide magnetic field:

$$
\begin{gathered}
\mathbf{B}_{\text {wiggler }}=\mathbf{B}_{w \perp}+\mathbf{B}_{w z} \\
\mathbf{B}_{\text {guide }}=B_{0} \hat{e}_{z}
\end{gathered}
$$

with

$$
\begin{aligned}
\mathbf{B}_{w \perp}= & 2 B_{w A} I_{1}^{\prime}\left(k_{w} R\right) \cos \left(\theta-k_{w} z\right) \hat{e}_{R} \\
& -2 B_{w A} \frac{I_{1}\left(k_{w} R\right)}{k_{w} R} \sin \left(\theta-k_{w} z\right) \hat{e}_{\theta}, \\
\mathbf{B}_{w z}= & 2 B_{w A} I_{1}\left(k_{w} R\right) \sin \left(\theta-k_{w} z\right) \hat{e}_{z},
\end{aligned}
$$

where $B_{0}$ is the amplitude of the guide magnetic field, $k_{w}=2 \pi / \lambda_{w}$ is the wiggler wave-number, $\lambda_{w}$ and $B_{w A}$ are the wiggler period and amplitude, $I_{1}$ and $I_{1}^{\prime}$ are the first kind of modified Bessel function and its derivative with respect to the whole argument, $\hat{e}_{R}, \hat{e}_{\theta}$ and $\hat{e}_{z}$ are the unit vectors in the radial, azimuthal and axial directions in a cylindrical coordinate system $(R, \theta, z)$, respectively. Suppose that pre-ionized plasma has been filled in the chamber. When the relativistic electron beam is injected in and passes through the chamber, it expels the electrons of the plasma to move outwardly, which leaves an ion core with a radius $R_{i o n}$, an average volume density $n_{i o n}$, and the ion-core electric field [24]

$$
\mathbf{E}_{i o n}=2 \pi e n_{i o n} \mathrm{Re}_{R}
$$

Meanwhile, the relativistic electron beam produces its electric field $\mathbf{E}_{\text {beam }}$ and magnetic field $\mathbf{B}_{\text {beam }}$ :

$$
\begin{gathered}
\mathbf{E}_{\text {beam }}=\left\{\begin{array}{ll}
-2 \pi e n_{b} \mathrm{Re}_{R}, & R<R_{b} \\
-2 \pi e n_{b} \frac{\mathrm{R}_{b}^{2}}{\mathrm{R}} \hat{\mathrm{e}}_{R}, & R \geqslant R_{b}
\end{array},\right. \\
\mathbf{B}_{\text {beam }}=\left\{\begin{array}{ll}
-2 \pi e n_{b} \frac{v_{z 0}}{c} \mathrm{R} \hat{\mathrm{e}}_{\varphi}, & R<R_{b} \\
-2 \pi e n_{b} \frac{v_{z 0}}{c} \frac{\mathrm{R}_{b}^{2}}{\mathrm{R}} \hat{\mathrm{e}}_{\varphi}, & R \geqslant R_{b}
\end{array},\right.
\end{gathered}
$$

where $R_{b}$ and $n_{b}$ are the electron-beam radius and volume density, respectively. Suppose that the input laser wave is a transverse-electric mode $\mathrm{TE}_{\mathrm{mn}}$ with its electric field $\mathbf{E}_{\text {laser }}$ and magnetic field $\mathbf{B}_{\text {laser }}$. Then, each individual electron in the relativistic electron beam is driven by all the electromagnetic forces, and can be described in terms of the equation of relativistic motion

$\frac{d \gamma m_{0} \mathbf{v}}{d t}=-e\left[\begin{array}{c}\left(\mathbf{E}_{\text {ion }}+\mathbf{E}_{\text {beam }}+\mathbf{E}_{\text {laser }}\right) \\ +\mathbf{v} \times\left(\mathbf{B}_{\text {wiggler }}+\mathbf{B}_{\text {guide }}+\mathbf{B}_{\text {laser }}\right)\end{array}\right]$,

where $\gamma=1 / \sqrt{1-v^{2} / c^{2}}$ is the relativistic energy factor, $e, m_{0}$ and $\mathbf{v}$ are the electron's charge, rest mass and velocity, and $c$ is the light speed in vacuum. The moving electron beam driven by the electromagnetic force has a current density $\mathbf{J}_{\text {beam }}=e n_{b} \mathbf{v}_{b}$. In turn, this current contributes to the laser field through the equation of wave

$$
\begin{aligned}
& \nabla^{2} \mathbf{A}_{\text {laser }}(R, \theta, z ; t)-\frac{1}{c^{2}} \frac{\partial^{2} \mathbf{A}_{\text {laser }}(R, \theta, z ; t)}{\partial t^{2}} \\
& =-\frac{4 \pi}{c} \mathbf{J}_{\text {beam }}(R, \theta, z ; t),
\end{aligned}
$$

where $\mathbf{A}_{\text {laser }}(R, \theta, z ; t)$ is the laser-wave vector potential which is defined by $\mathbf{E}_{\text {laser }}=-\frac{1}{c} \frac{\partial}{\partial t} \mathbf{A}_{\text {laser }}(R, \theta, z ; t)$ and $\mathbf{B}_{\text {laser }}=\nabla \times \mathbf{A}_{\text {laser }}(R, \theta, z ; t)$.

In nonlinear simulations, the equation of wave (9) is coupled with the equation of motion (8) by discretizing the electron beam into test electrons. In this way the differential vector equation of order two, equation (9), can be rewritten into the following four differential scalar equations of order one:

$$
\begin{gathered}
\frac{d A(z)}{d z}=A(z) G(z), \\
\frac{d \varphi(z)}{d z}=\varphi^{\prime}(z), \\
\frac{d G(z)}{d z}=\left[k_{z}+\varphi^{\prime}(z)\right]^{2}-k_{z}^{2}+\frac{S(z)}{A(z)}-G^{2}(z), \\
\frac{d \varphi^{\prime}(z)}{d z}=\frac{T(z)}{A(z)}-2 G(z)\left[k_{z}+\varphi^{\prime}(z)\right],
\end{gathered}
$$

where $G(z)$ is the gain of the laser wave, which is defined as

$$
G(z)=\frac{1}{A(z)} \frac{d A(z)}{d z}
$$

and

$$
\begin{aligned}
S(z)= & \frac{-8 I_{b}}{N K_{m n} c} \sum_{j=1}^{N} \frac{1}{v_{z j}}\left[v_{\theta j} k_{c} J_{m}^{\prime}\left(k_{c} r_{j}\right) \cos \Phi_{j}\right. \\
& \left.+v_{r j} \frac{m}{R_{j}} J_{m}\left(k_{c} r_{j}\right) \sin \Phi_{j}\right], \\
T(z)= & \frac{8 I_{b}}{N K_{m n} c} \sum_{j=1}^{N} \frac{1}{v_{z j}}\left[v_{\theta j} k_{c} J_{m}^{\prime}\left(k_{c} r_{j}\right) \sin \Phi_{j}\right. \\
& \left.-v_{r j} \frac{m}{r_{j}} J_{m}\left(k_{c} r_{j}\right) \cos \Phi_{j}\right], \\
\Phi_{j}= & k_{z} z-\omega t_{j}+m \theta_{j}+\varphi_{j}(z), \\
& K_{m n}=k_{c}^{2} R_{w}^{2} J_{m}^{2}\left(k_{c} R_{w}\right) .
\end{aligned}
$$


Table 1. Output power versus the ion-channel parameter.

\begin{tabular}{ll}
\hline Ion-channel parameter & Output power (MW) \\
\hline 0.00 & 51.58 \\
0.03 & 52.84 \\
0.05 & 53.59 \\
0.07 & 54.25 \\
0.10 & 55.04 \\
0.13 & 55.60 \\
0.15 & 55.84 \\
0.17 & 56.01 \\
0.20 & 56.13 \\
0.23 & 56.18 \\
0.25 & 56.22 \\
\hline
\end{tabular}

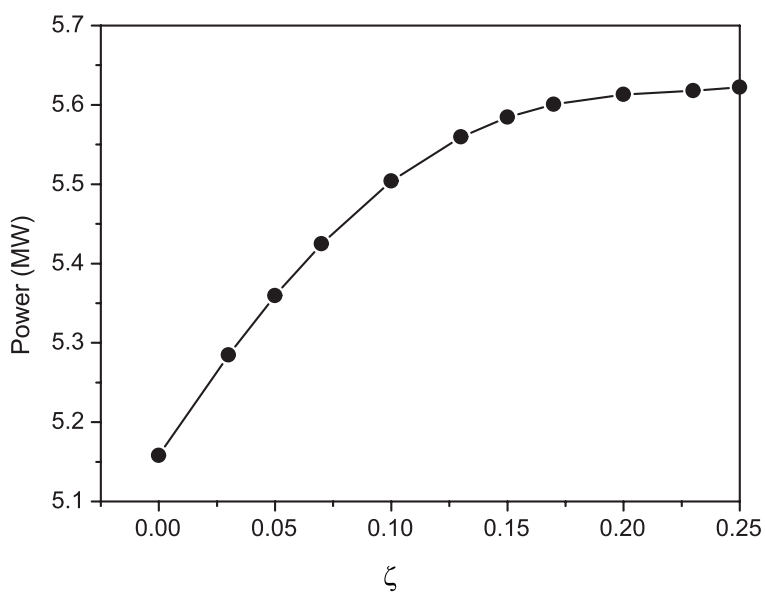

Figure 1. Output power versus the ion-channel parameter $\varsigma$ in a Raman FEL with an ion-channel, where the design parameters in the MTT experiment [20] are used.

In the above equations, $N$ is the total number of individual test electrons; $R_{w}$ is the radius of the cylindrical waveguide; $m, n$, $\omega, k_{c}, k_{z}$, and $\varphi(z)$ denote, respectively, the azimuthal modeindex, radial mode-index, angular frequency, cutoff wavenumber, axial wave-number, and phase of the $T E_{m, n}$-mode wave; $J_{m}$ and $J_{\mu}^{\prime}$ are the first kind of Bessel function of order $m$ and their derivatives with respect to the whole argument. In the above wave equations, the independent variable, time $t$, has been transferred to $z$ by the relationship $d / d t=v_{z} d / d z$.

Coupling the laser wave equations (10)-(13) with the equation of motion (8) gives a set of self-consistent differential equations of the wave-beam interaction, and the laser field can be solved. Then, evolution of the laser power along the longitudinal position in the chamber as well as the output power at the exit can be understood with the following expression

$$
P_{\text {laser }}=\frac{\omega}{8} K_{m n}\left[k_{z}+\varphi^{\prime}(z)\right] A^{2}(z) .
$$

\section{Power enhancement}

According to the coupling differential equations (8), (10)(13), a code is programmed for the nonlinear simulations. Without loss of generality, here we take the MIT experiment as an example to show how the output power may be

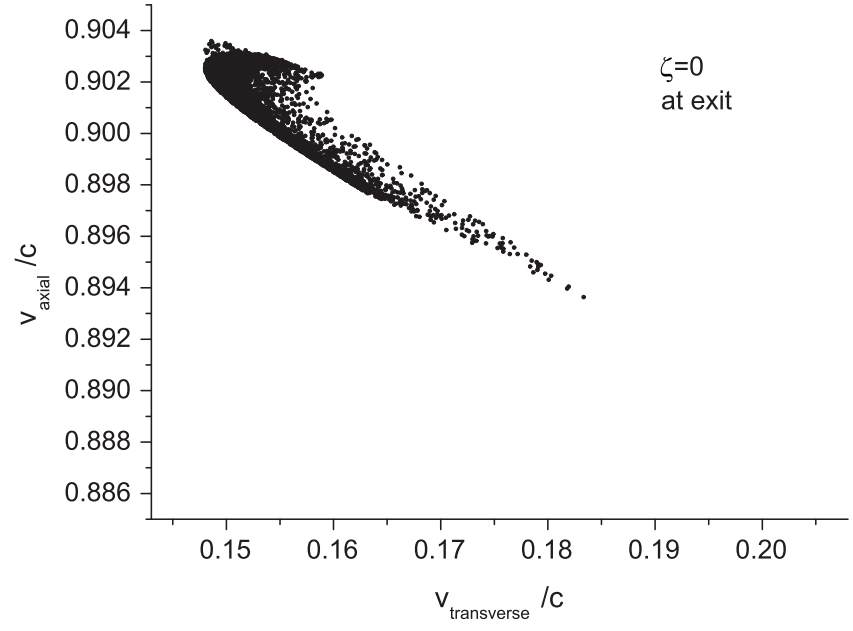

(a)

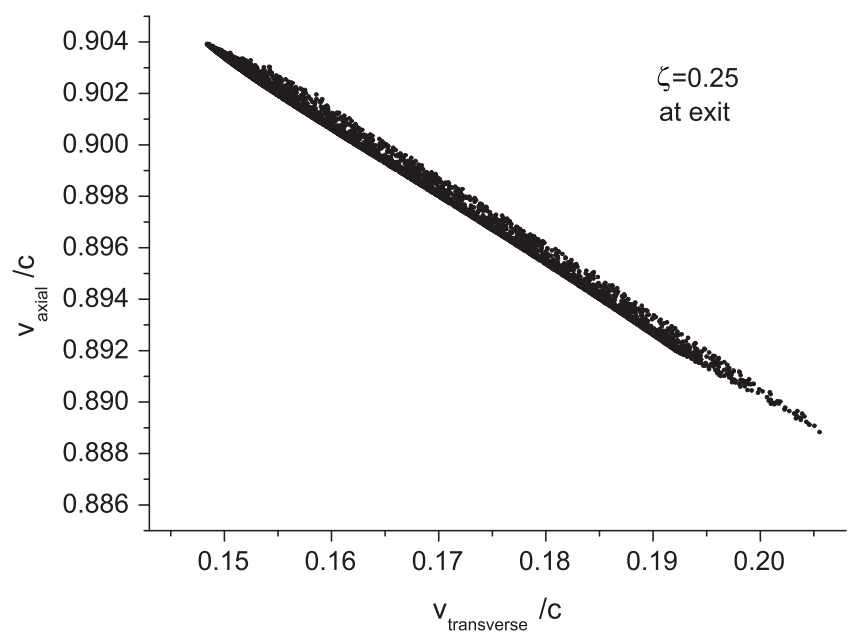

(b)

Figure 2. Distribution of the normalized transverse and longitudinal velocity of the test electrons at the exit of the chamber (a) without an ion-channel $(\zeta=0)$, and (b) with an ion-channel $(\zeta=0.25)$.

enhanced by applying an ion-channel. In the MIT experiment, the electron-beam current, energy, energy spread, and radius were $300 \mathrm{~A}, 750 \mathrm{keV}, 1 \%$, and $0.25 \mathrm{~cm}$; the length and radius of the cylindrical waveguide were $2 \mathrm{~m}$ and $0.51 \mathrm{~cm}$; the bifilar helical wiggler field had a period of $3.18 \mathrm{~cm}$, and its amplitude was slowly increased over the initial six periods up to $630 \mathrm{G}$; an axial guide magnetic field was $4060 \mathrm{G}$; a TE 11 -mode laser wave at frequency of $33.39 \mathrm{GHz}$, with an effective incident power of $8.5 \mathrm{~kW}$ was put in to participate in the FEL interaction [20]. We first examined the credibility of our code and got a simulated output power of 5.53 MW at the exit, which was in good agreement with the reported measurement of 5.8 MW [20].

Then, we examined how the output power of the laser wave was affected by applying an ion-cannel. For the convenience of comparison, we define an ion-channel parameter $\varsigma=n_{\text {ion }} / n_{b}$, where $\zeta=0$ means no ion-channel was applied, which corresponds to the situation of the MIT experiment [20]. In the simulations we found the wave power approached saturation at the distance of $70 \mathrm{~cm}$, and so, without loss of 
generality, we truncated the calculation at that position to save CPU. This treatment does not deteriorate the practical significance because the shorter beam-wave interaction length corresponds to a shorter wiggler and can cut down the manufacture cost of the facility. Table 1 lists the output power versus the ion-channel parameter, and the corresponding data are plotted in figure 1. It is clearly shown that the output power goes up with application of the ion-channel, and gets an increase of $9 \%$ when the ion-channel parameter is $\zeta=0.25$.

\section{Physical examination}

The effect of the ion-channel on the enhancement of the output power may be partly explained as the improvement of the electron-beam transportation. Since the electric field produced by the ion hose of the ion-channel must affect the dynamic behavior of all the individual electrons, in simulations we also traced the motion of the 5000 test electrons, and compared the situations with and without an ion-channel. Figure 2 plots the normalized transverse and longitudinal velocities of the test electrons at the exit of the beam-wave interaction region for the situations without the ion-channel $(\zeta=0)$ and with the ion-channel $(\zeta=0.25)$. It can be seen that the electrons in the former span a wider range than in the latter, which means the electron beam without the ion-channel $(\zeta=0)$ may get a greater velocity spread than that with the ion-channel $(\zeta=0.25)$. As is well known, the velocity spread deteriorates the quality of the electron-beam transportation and decreases the effectiveness of the beam-wave interaction. Therefore, application of an adjusted ion-channel leads to a better electron-beam transportation quality and thus results in a higher output power than that without an ion-channel.

Meanwhile, it is worthy to note that the improvement of the output power in a Raman free-electron laser by applying an ionchannel is finite. The reason for this is that the dynamic behavior is governed not only by the ion-channel field but also by the wiggler field, the guide magnetic field, and the laser field. This fact explains why the curve of the power enhancement versus the ion-channel parameter gradually becomes flat in figure 1 .

\section{Conclusions}

In this paper, output power in a Raman free-electron laser in a millimeter wave range with and without the application of an ion-channel is studied by credible simulations. Results reveal that the power enhancement may be realized by applying an adjusted ion-channel in the beam-wave interaction chamber, and at least a power enhancement of $9 \%$ could be expected. Results also show that the mechanism of the power enhancement-by applying an ion channel-lies in the fact that the ion-channel field decreases the velocity's spread of the electron beam, and thus improves the electron-beam transportation quality.

\section{Acknowledgments}

This work was partly supported by the ChunHui-Plan Project of Ministry of Education, China (Grant No. z2011089), and the Graduate Innovation Fund of Xihua University, China (Grant No. ycjj2017061).

\section{References}

[1] Guetg M, Lutman A, Ding Y, Maxwell T, Decker F, Bergann U and Huang Z 2018 Phys. Rev. Lett. 120014801

[2] Kang H et al 2017 Nat. Photon. 11 708-13

[3] Preston T et al 2017 Phys. Rev. Lett. 119085001

[4] Toufarova M et al 2017 Phys. Rev. B 96214101

[5] Kluge Tet al 2017 Phys. Plasmas 24102709

[6] Gunst J, Wu Y, Kumar N, Keitel C and Palffy A 2015 Phys. Plasmas 22112706

[7] Vinko S 2015 J. Plasma Phys. 81365810501

[8] Eliasson B and Liu C 2013 J. Plasma Phys. 79 995-8

[9] Emma P et al 2012 Nat. Photon. 4641

[10] Khan S 2008 J. Mod. Opt. 553469

[11] Marshall T C 1985 Free-Electron Lasers (New York: Macmillan)

[12] Brau C A 1990 Free-Electron Lasers (Cambridge, MA: Academic)

[13] Luchini P and Motz H 1990 Undulators and Free-Electron Lasers (Oxford: Clarendon)

[14] Dattoli G, Renieri A and Torre A 1993 Lectures on the Free Electron Laser Theory and Related Topics (London: World Scientific)

[15] Zhang S-C 1993 Introduction to Free-Electron Lasers (Chengdu: SWJTU Press)

[16] Freund H P and Antosen T M Jr 1996 Principles of Free-Electron Lasers (New York: Chapman and Hall)

[17] Saldin E L, Schneidmiller E A and Yurkov M V 1999 The Physics of Free Electron Lasers (New York: Springer)

[18] Zhang S-C and Thumm M 2010 Phys. Lett. A 3741745

[19] Freund H, Johnston S and Sprangle P 1983 IEEE J. Quantum Electron. 19322

[20] Conde M and Bekefi G 1991 Phys. Rev. Lett. 673082

[21] Huang X-L, Wang S-J, Xu Y-G and Zhang S-C 2012 Phys. Rev. ST Accel. Beams 15120702

[22] Zhang S-C 2013 Phys. Lett. A 377319

[23] Takayama K and Hiramatsu S 1988 Phys. Rev. A 37173

[24] Whittum D 1990 DhD dissertation University of California at Berkeley ch 2

[25] Whittum D and Sessler A M 1990 Phys. Rev. Lett. 642511

[26] Ebihara O et al 1992 Nucl. Instrum. Methods Phys. Res. A 318101

[27] Whittum D et al 1993 IEEE Trans. Plasma Sci. 21136

[28] Jha P and Kumar P 1998 Phys. Rev. E 572256

[29] Fu W and Yan Y 2008 Int. J. Infrared Millim. Waves 2943

[30] Saviz S, Rezael Z and Aghamir F 2012 Phys. Plasmas 19203115

[31] Mehdian H, Alimohamadi M and Hasanbeigi A 2012 J. Plasma Phys. 78537

[32] Esmaeilzadeh M and Taghavi A 2012 Phys. Plasmas 19113101

[33] Bahman F and Maraghechi B 2013 Phys. Plasmas 20023101

[34] Hasanbeigi A, Abasirostami S and Mehdian H 2013 J. Plasma Phys. 79 853-7

[35] Ouyang Z and Zhang S-C 2014 Laser Phys. 24105002

[36] Ersfeld B, Bonifacio R, Chen S, Islam M, Smorenburg P and Jaroszynski D 2014 New J. Phys. 16093025

[37] Mohsenpour T and Alirezaee H 2014 Phys. Plasmas 21082113

[38] Kheiri G and Esmaeilzadeh M 2014 Phys. Scr. 89125603

[39] Ouyang Z and Zhang S-C 2015 Phys. Plasmas 22043111

[40] Xu J et al 2015 J. Plasma Phys. 81905810506

[41] Amri H and Mohsenpour T 2016 Phys. Plasmas 23022101 